\title{
A Pragmatic Approach to Impairment Awareness in Optical Networks
}

\author{
Farabi Iqbal \\ Network Architectures and Services, \\ Delft University of Technology, \\ Mekelweg 4, 2628 CD Delft, \\ The Netherlands \\ Email: M.A.F.Iqbal@tudelft.nl
}

\author{
Rob Smets \\ SURFnet, \\ Moreelsepark 48, 3511 EP Utrecht, \\ The Netherlands, \\ Email: Rob.Smets@surfnet.nl
}

\author{
Fernando A. Kuipers \\ Network Architectures and Services, \\ Delft University of Technology, \\ Mekelweg 4, 2628 CD Delft, \\ The Netherlands \\ Email: F.A.Kuipers@tudelft.nl
}

\begin{abstract}
The points-of-presence of optical networks are interconnected by photonic paths capable of carrying Terabits of data. However, signals along those photonic paths accumulate transmission impairments and thus can be unreadable at the receiver if the accumulated impairments are too high. Our contributions in this paper are three-fold: (1) we propose the use of a realistic link structure to quantify the potential harm of impairments, (2) we propose a new additive routing metric, referred to as the Figure-of-Impact (FoI), that quantifies the potential harm of the non-linear Self-Phase Modulation (SPM) impairment, and (3) we propose a two-phase heuristic for solving the multi-constrained impairment-aware routing and wavelength assignment problem and apply it to a realistic network.
\end{abstract}

\section{INTRODUCTION}

A photonic path is a preconfigured transmission path on a single wavelength channel between two network nodes. In the absence of electronic regeneration [1], signals accumulate transmission impairments [2] over their photonic path and become unusable when those accumulated impairments surpass the tolerable limit at the destination node. Linear impairments (e.g., loss, noise and dispersion) are independent of the signal power and affect each wavelength individually. On the other hand, non-linear impairments (e.g., Self-Phase Modulation (SPM) and Cross-Phase Modulation (XPM)) depend on the signal power, and may cause disturbance and interference between wavelengths. Quantifying the negative effects of impairments usually requires complex procedures that are computationally intensive. For instance, a high fidelity network simulator could take days to compute a path when considering impairments. In some cases, such as service restoration upon simultaneous failure of both the primary and backup paths of a connection, time-consuming computation will incur additional service penalty to the network administrator. We propose a fast, pragmatic approach that quantifies the potential harm (instead of a high fidelity calculation) of impairments in a realistic network setting.

Often, a simplified and rigid link structure is used to represent network links (e.g., [1], [3] consider a link as a single optical fiber, [4] considers a link as an optical fiber with attached amplifiers at each end, and [5] considers a link as a concatenation of a multiplexer, an amplifier, a fiber span, an amplifier and a demultiplexer, in that order, all of which are not representative enough for practical use). In realistic networks, different links comprise different network equipment with different impairment effects. In Section II, we consider a link as a concatenation of different link blocks (each corresponding to a network equipment), and quantify the potential harm of impairments at each link block.

In Section III, we propose a new metric referred to as the Figure-of-Impact (FoI) to represent the potential harm of SPM. The FoI enables the consideration of the non-linear SPM impairment as an additive routing metric, which is useful in finding feasible photonic paths, without needing to estimate the SPM effect of each link as part of the link metric before finding paths as done in [6], [7].

In Section IV, we propose a two-phase heuristic for solving the multi-constrained impairment-aware routing and wavelength assignment problem. We consider multiple impairment constraints, instead of only a single impairment as done in [4]. Contrary to [1], [3], [4], [5] which consider unidirectional connections, we consider bidirectional connections (each with a feasible photonic path from the source node to the destination node, and another feasible photonic path from the destination node to the source node).

\section{LINK STRUCTURE}

We propose the link structure illustrated in Figure 1. Each network node is equipped with an optical add-drop multiplexer (OADM) or a reconfigurable optical add-drop multiplexer (ROADM) [8]. (R)OADMs add, drop and/or optically pass signals. Each directed link consists of a concatenation of link blocks. A directed link from node $u$ to node $v$ can have link blocks that are different from the directed link from node $v$ to node $u$. Four types of link blocks are considered in this paper,

1) Fiber block - represents an optical fiber span (e.g., Non Dispersion-Shifted Fiber (NDSF) or Non-Zero Dispersion-Shifted Fiber (NZDSF)).

2) Amplifier block - represents an optical amplifier.

3) DSCM block - represents a Dispersion Slope Compensating Module (DSCM).

4) Loss block - represents an attenuator, non-amplifier line interface module, connector transitions, or any other equipment that produces loss. 


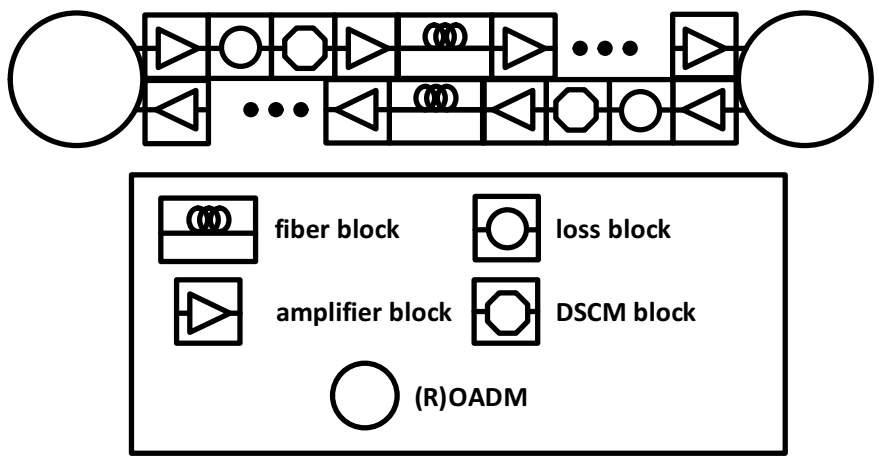

Fig. 1. An example of a link structure.

Our link structure enables the quantification of various signal parameters at each link block during path computation. For example, we consider four types of signal parameters in this paper, namely power level, dispersion, Figure of Merit (FoM) [4], and Figure of Impact (FoI) (explained later in Section III). We also address XPM in Section IV.

\section{A. Power Level}

The power level of signals is gradually attenuated along their photonic path due to the loss at (R)OADMs, fiber blocks, DSCM blocks and loss blocks. To compensate these losses, amplifier blocks are placed at strategic points along the link to boost the signal power to its initial power level.

(R)OADM loss - The signal power is reduced by the insertion loss $\alpha_{s}^{\text {in }}$ of the source node (R)OADM, the pass-through loss $\alpha_{n}^{\text {pass }}$ of the (R)OADM of each intermediate node $n$, and the outgoing loss $\alpha_{t}^{\text {out }}$ of the destination node (R)OADM.

Fiber block loss - The fiber block $f$ reduces signal power by $\alpha_{f} \ell_{f}$, where $\alpha_{f}$ is the fiber loss coefficient in $\mathrm{dB} / \mathrm{km}$, and $\ell_{f}$ is the fiber length in $\mathrm{km}$.

DSCM block loss - The DSCM block $d$ reduces signal power by $\left(1.7+0.06 \times \ell_{d}\right)$, where $\ell_{d}$ is the DSCM length in $\mathrm{km}$.

Loss block loss - The loss block $l$ reduces signal power by the equipment loss, e.g., $1 \mathrm{~dB}$ loss for non-amplifier line interface modules, $0.5 \mathrm{~dB}$ loss for connector transitions, or the loss of attenuators. Attenuators ensure that the signal power is kept below a certain level to satisfy the minimum amplifier, and avoid a more serious SPM effect due to high signal power.

Amplifier block gain - The amplifier block $a$ boosts signal power to its launch power (e.g., $10 \mathrm{Gbps}$ signals have a $0 \mathrm{dBm}$ typical launch power, while $100 \mathrm{Gbps}$ signals have a $3 \mathrm{dBm}$ typical launch power). We limit the number of signals that traverse each $a$, so that its total output power does not exceed its power saturation limit $P_{s a t}$. Above $P_{s a t}$, the amplifier gain reduces, affecting the signal's output power and noise.

\section{B. Dispersion}

Dispersion reduces signal power within the bit slot, and spreads the signal power beyond the allocated bit slot, leading to inter-symbol interference. Dispersion occurs due to the fiber block, but can be compensated by the DSCM block.

Fiber block dispersion - The fiber block $f$ increases signal dispersion by $\gamma_{f} \ell_{f}$, where $\gamma_{f}$ is the fiber dispersion coefficient in $\mathrm{ps} / \mathrm{nm} \cdot \mathrm{km}$, and $\ell_{f}$ is the fiber length in $\mathrm{km}$.

DSCM block compensation - The DSCM block $d$ reduces signal dispersion by $\beta_{d} \ell_{d}$, where $\beta_{d}$ is the DSCM compensation coefficient in $\mathrm{ps} / \mathrm{nm} \cdot \mathrm{km}$, and $\ell_{d}$ is the DSCM length in $\mathrm{km}$.

\section{Figure-of-Merit (FoM)}

Though amplifiers are useful for compensating loss, they introduce Amplifier Spontaneous Emission (ASE) noise. Beshir et al. [4] proposed the Figure-of Merit (FoM) metric to represent the potential harm of noise. We extend the use of FoM to adapt to our link structure. The FoM between two amplifier blocks $a_{i}$ and $a_{j}$ can be computed as,

$$
\mathrm{FoM}=10^{\frac{L_{i j}}{10}}
$$

where $L_{i j}$ is the total loss in $\mathrm{dB}$ between $a_{i}$ and $a_{j}$. The FoM of a photonic path is the sum of the FoMs between each consecutive amplifier in the photonic path.

\section{Figure-of-Impact (FoI)}

In Section III, we introduce a new metric referred to as the Figure-of-Impact (FoI) for quantifying the potential harm of SPM. The total FoI of a photonic path is computed as the sum of the FoI at points along the path where the signal dispersion is zero. Examples of possible zero dispersion points are at the beginning of the photonic path, within the DSCM block and within the fiber block. At these points, the signal power is computed and the FoI is computed by Equation 13.

\section{E. Computation example}

Consider a 10 Gbps Amplitude-Shift Keying (ASK) signal that uses a photonic path $p$ of wavelength $1530 \mathrm{~nm}$ from node 1 to node 3 via node 2 as illustrated in Figure 2. All the parameters required for computing the total path dispersion $\left(D_{p}\right)$, the total path FoM $\left(\mathrm{FoM}_{p}\right)$, and the total path FoI $\left(\mathrm{FoI}_{p}\right)$ are shown in Table I.

$$
\begin{aligned}
D_{p}= & D_{1}+D_{2}+D_{3}-D_{4}+D_{5}+D_{6}-D_{7}+D_{8}-D_{9} \\
= & \gamma_{1} \ell_{1}+\gamma_{2} \ell_{2}+\gamma_{3} \ell_{3}-\beta_{4} \ell_{4}+\gamma_{5} \ell_{5}+\gamma_{6} \ell_{6}-\beta_{7} \ell_{7} \\
& +\gamma_{8} \ell_{8}+\gamma_{9} \ell_{9} \\
= & 604.65 \mathrm{ps} / \mathrm{nm}
\end{aligned}
$$

$$
\begin{aligned}
\mathrm{FoM}_{p}= & \mathrm{FoM}_{1}+\mathrm{FoM}_{2}+\mathrm{FoM}_{3}+\mathrm{FoM}_{4}+\mathrm{FoM}_{5}+\mathrm{FoM}_{6} \\
& +\mathrm{FoM}_{7}+\mathrm{FoM}_{8} \\
= & 694.991
\end{aligned}
$$

$$
\begin{aligned}
\mathrm{FoI}_{p} & =\mathrm{FoI}_{1}+\mathrm{FoI}_{2}+\mathrm{FoI}_{3} \\
& =\nu P_{1}+\nu P_{2}+\nu P_{3} \\
& =0.118
\end{aligned}
$$




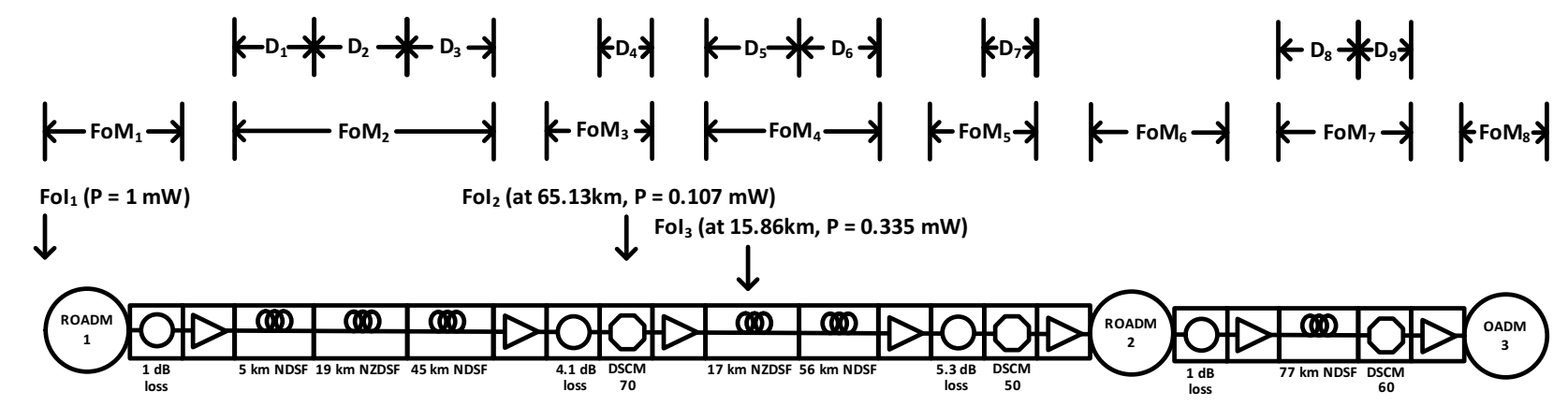

Fig. 2. An example of a photonic path.

TABLE I

SIMULATION PARAMETERS.

\begin{tabular}{|c|c|c|}
\hline Parameter & Symbol & Value \\
\hline \hline Amplifier maximum output power & $P_{\text {sat }}$ & $20 \mathrm{dBm}$ \\
\hline DSCM compensation coefficient & $\beta_{d}$ & $13.15 \mathrm{ps} / \mathrm{nm} \cdot(1530 \mathrm{~nm}), 14.95 \mathrm{ps} / \mathrm{nm} \cdot \mathrm{km}(1565 \mathrm{~nm})$ \\
\hline NDSF dispersion coefficient & $\gamma_{f}$ & $15.55 \mathrm{ps} / \mathrm{nm} \cdot \mathrm{km}(1530 \mathrm{~nm}), 17.78 \mathrm{ps} / \mathrm{nm} \cdot \mathrm{km}(1565 \mathrm{~nm})$ \\
\hline NZDSF dispersion coefficient & $\gamma_{f}$ & $3.5 \mathrm{ps} / \mathrm{nm} \cdot \mathrm{km}(1530 \mathrm{~nm}), 5.12 \mathrm{ps} / \mathrm{nm} \cdot \mathrm{km}(1565 \mathrm{~nm})$ \\
\hline NDSF and NZDSF effective core area & $A_{e f f}$ & $80 \mu \mathrm{m}^{2}$ \\
\hline NDSF and NZDSF loss coefficient & $\alpha_{f}$ & $0.25 \mathrm{~dB} / \mathrm{km}(\mathrm{or} 31.66 \mu \mathrm{Np} / \mathrm{m})$ \\
\hline NDSF and NZDSF non-linear refractive index coefficient & $\eta_{2}$ & $3.2 \times 10^{-20} \mathrm{~m}^{2} / \mathrm{W}[9]$ \\
\hline Initial signal power & $P_{\text {in }}$ & $0 \mathrm{dBm}(10 \mathrm{Gbps} \mathrm{signal}), 3 \mathrm{dBm}(100 \mathrm{Gbps}$ signal $)$ \\
\hline (R)OADM insertion loss & $\alpha_{1}^{\text {in }}$ & $10 \mathrm{~dB}(\mathrm{OADM}), 16 \mathrm{~dB}(\mathrm{ROADM})$ \\
\hline (R)OADM outgoing loss & $\alpha^{\text {out }}$ & $6 \mathrm{~dB}(\mathrm{OADM}), 10 \mathrm{~dB}(\mathrm{ROADM})$ \\
\hline (R)OADM pass-through loss & $\alpha^{\text {pass }}$ & varies between $6 \mathrm{~dB}$ and $16 \mathrm{~dB}$ based on nodes \\
\hline Signal dispersion constraint & $D_{\max }$ & must be between -400 and $1600 \mathrm{ps} / \mathrm{nm}(10 \mathrm{Gbps})$ \\
\hline Signal FoM constraint & FoM & $720(10 \mathrm{Gbps}), 900(100 \mathrm{Gbps})$ \\
\hline Signal Fol constraint & FoI max & $0.4(10 \mathrm{Gbps}$ and $100 \mathrm{Gbps})$ \\
\hline
\end{tabular}

\section{The FiguRE-OF-IMPACT (FoI)}

Self-Phase Modulation (SPM) occurs due to the Kerr effect, a variation of the fiber refractive index over distance to the signal power. Different optical pulse parts undergo different phase shift, chirping the pulse. The spectral broadening due to SPM contributes to greater temporal broadening due to dispersion. SPM is a concern for signals operating at $10 \mathrm{Gbps}$ or more, especially at high signal power.

We introduce a new metric referred to as the Figure-ofImpact (FoI) for quantifying the potential harm of SPM. FoI represents the ratio of the non-linear phase shift due to SPM to the linear phase shift due to dispersion. The total $\mathrm{FoI}_{p}$ of a photonic path $p$ is computed as the sum of the FoI at points along the path where dispersion $D$ is fully compensated,

$$
\mathrm{FoI}_{p}=\sum_{j=1, \ldots, J} \mathrm{FoI}_{j} \quad \mid D_{j}=0
$$

where $J$ is the number of times the dispersion becomes zero in the photonic path. The more frequently a signal is fully dispersion compensated, and the higher the signal power is at these points, the higher the total FoI of the photonic path FoI $p$ will be. The accumulated phase shift $\phi$ of a signal over a transmission distance $z$ can be expressed as,

$$
\begin{aligned}
\phi & =\frac{2 \pi}{\lambda} \eta_{\text {eff }} \ell_{e f f} \\
& =\frac{2 \pi}{\lambda}\left(\eta_{0}+\eta_{2} I_{z}\right) \ell_{e f f} \\
& =\frac{2 \pi}{\lambda} \eta_{0} \ell_{e f f}+\frac{2 \pi}{\lambda} \eta_{2} I_{z} \ell_{e f f}
\end{aligned}
$$

where $\eta_{\text {eff }}$ is the fiber effective refractive index coefficient, $\eta_{0}$ is the fiber linear refractive index coefficient, $\eta_{2}$ is the fiber non-linear refractive index coefficient, $I_{z}$ is the pulse intensity at distance $z$ and $\ell_{\text {eff }}$ is the effective fiber length where the signal power is assumed to be constant up to that length.

$I_{z}$ can be computed as,

$$
I_{z}=\frac{P_{z}}{A_{\text {eff }}}=\frac{P_{l} e^{-\alpha_{f} z}}{A_{\text {eff }}}
$$

where $P_{z}$ is the signal power in $W$ at distance $z, P_{l}$ is the optical launch power, $A_{e f f}$ is the effective fiber core area (i.e., the size of the area where optical power is confined within the fiber core), and $\alpha_{f}$ is the fiber loss coefficient.

$\ell_{e f f}$ can be computed as,

$$
\ell_{e f f}=\int_{0}^{\ell} e^{-\alpha l} d l=\frac{1}{\alpha_{f}}\left(1-e^{-\alpha \ell}\right)
$$


where $\ell$ is the fiber length. For a long fiber, $\ell_{e f f} \sim \frac{1}{\alpha_{f}}$.

The right part of the phase shift $\phi$ is the non-linear phase shift $\phi_{N L}$ due to SPM. $\phi_{N L}$ can thus be expressed as,

$$
\begin{aligned}
\phi_{N L} & =\frac{2 \pi}{\lambda} \eta_{2} \frac{P_{z}}{A_{e f f}} \ell_{e f f} \\
& \sim \frac{2 \pi}{\lambda} \frac{\eta_{2}}{\alpha_{f} A_{e f f}} P_{z}
\end{aligned}
$$

The corresponding amount of chirp $\Delta \omega_{N L}$ due to $\phi_{N L}$ is,

$$
\Delta \omega_{N L}=\frac{\partial \phi_{N L}}{\partial t}=\frac{2 \pi}{\lambda} \frac{\eta_{2}}{\alpha_{f} A_{\text {eff }}} \frac{\partial P_{z}}{\partial t}
$$

We can use a Taylor-based expansion to convert changes in optical frequency to a change in optical wavelength for obtaining a measure for the amount of power that is dispersed in time as the cause of Inter-Symbol Interference. The impact of fiber non-linearity can then be expressed as a time interval in which the signal power is dispersed,

$$
\Delta \tau_{N L}=D \ell \frac{-\lambda^{2}}{c} \Delta \omega_{N L}=-D \ell \frac{2 \pi \lambda}{c} \frac{\eta_{2}}{\alpha_{f} A_{e f f}} \frac{\partial P_{z}}{\partial t}
$$

Assume that $P_{z}$ is a raised cosine at the symbol rate of the modulating signal. In order to evaluate the maximum dispersion induced time shift, the derivative of a typical signal $P_{z}$ with respect to time is needed. In general, the largest frequency component that can be expected in the signal is the frequency that corresponds to half the symbol rate $B$,

$$
P_{z}=\frac{1}{2} P_{l}(1+\sin (\pi B \tau))
$$

The maximum value of the derivative is

$$
\begin{gathered}
\frac{\partial p_{z}}{\partial t}=\frac{1}{2} P_{l} \pi B \\
\Delta \tau_{N L}=-D \ell \frac{2 \pi^{2} \lambda}{c} \frac{\eta_{2}}{\alpha_{f} A_{e f f}} P_{l} B
\end{gathered}
$$

Equation 11 indicates the time shift experienced by a signal when propagating across a distance $z$ caused by a launch power $P_{l}$ where the signal itself is not assumed to become dispersed. Hence, dispersion is not a mechanism that reduces the non-linear phase shift. If we consider this, it can be seen that the non-linear phase shift adds to the time shift resulting only from dispersion $\Delta \tau_{d}$ already present in the fiber,

$$
\Delta \tau_{d}=-D \ell \frac{\lambda^{2}}{c} B
$$

Let us determine the ratio of these two time shifts, which we shall refer to as the Figure of Impact (FoI),

$$
\mathrm{FoI}=\frac{\Delta \tau_{N L}}{\Delta \tau_{d}}=\frac{\pi^{2}}{\lambda} \frac{\eta_{2}}{\alpha_{f} A_{\text {eff }}} P_{l}=\nu P_{l}
$$

It becomes clear that the more power is launched into the fiber the higher the FoI will be. For instance, a power level of $5 \mathrm{~mW}$ which corresponds to a FoI of 0.40 will start to deteriorate NRZ signals in an observable way, and should be avoided.

\section{APPLICATION}

We define the Multi-constrained Impairment-aware Routing and Wavelength Assignment (MIRWA) problem:

MIRWA problem - Given a directed graph $G=(\mathcal{N}, \mathcal{E})$ consisting of a set $\mathcal{N}$ of $N$ nodes and a set $\mathcal{E}$ of $E$ links, a set $\mathcal{R}$ of $R$ bidirectional connection requests, and an impairment constraint set $\mathcal{I}=\left\{D_{\max }, \mathrm{FoM}_{\max }, \mathrm{FoI}_{\max }\right\}$. Each unidirectional link $(u, v) \in \mathcal{E}$ that connects node $u$ to node $v$ is associated with a set $\mathcal{L}$ of $L$ link blocks, and a set $\mathcal{Y}$ of $Y$ wavelengths. Each request $r \in \mathcal{R}$ is associated with a tuple $\left(s_{r}, t_{r}, \delta_{r}\right)$, where $s_{r}$ is the source node, $t_{r}$ is the destination node and $\delta_{r}$ is the transmission rate. Assign each $r \in \mathcal{R}$ with two photonic paths (from $s_{r}$ to $t_{r}$, and from $t_{r}$ to $s_{r}$ ) of a wavelength $\lambda \in \mathcal{Y}$ that satisfy $\mathcal{I}$, such that $Y$ is minimized.

The MIRWA problem is NP-hard since it is composed of two NP-hard problems, namely the routing with multiple constraints problem [10], and the routing and wavelength assignment problem [11]. Hence, we develop a two-phase heuristic for the MIRWA problem. Phase 1 solves the multiconstrained impairment aware routing problem exactly, and Phase 2 solves the wavelength assignment problem exactly.

\section{A. Phase 1}

In Phase 1, we propose the Multi-constrained Impairmentaware Routing (MIR) algorithm that is based on the $k$-shortest paths approach [12], [13] to find at most $K$ bidirectional connections for each $r \in \mathcal{R}$ that satisfy $\mathcal{I}$ at a given $\lambda \in \mathcal{Y}$. The MIR algorithm can also be used independently to find a single connection $(K=1)$ for path restoration purposes. In the context of the MIRWA problem, we use the minimum wavelength $(\lambda=1530 \mathrm{~nm})$ as an input to the MIR algorithm, and also ensure that the returned connections are also feasible at the maximum wavelength $(\lambda=1565 \mathrm{~nm})$ in line 8 of the MIR algorithm. Hence, all the $K$ connections of each $r \in \mathcal{R}$ will satisfy $\mathcal{I}$ at all possible wavelengths in the set $\mathcal{Y}$.

The pseudocode of the MIR algorithm is given in Algorithm 1. Each node $n$ will keep at most $k$ subpaths, with each subpath denoted by $p_{n k}$. A set of subpaths maintained by $n$ is denoted by $p_{n}$. Each $p_{n k}$ is paired with its last visited node, total hop count $h_{n k}$, latest signal power $P_{n k}$, total dispersion $D_{n k}$, total loss since last amplifier $L_{n k}$, total $\mathrm{FoM}_{n k}$ and total $\mathrm{FoI}_{n k}$.

A subpath consisting of only the source node $p_{s 1}$ is initialized in lines 1-2, and inserted into the queue $Q$ in line 3. While $Q$ contains at least a subpath, the subpath with the lowest $h_{n k}$ is extracted from $Q$ as $p_{u k}$ in line 5. If the last visited node of $p_{u k}$ is $t$, and $\mathcal{I}$ are satisfied by both $p_{u k}$ and the reverse subpath $p_{u k}^{\prime}, p_{u k}$ is recorded as a feasible connection in line 9. When $K$ feasible connections have been recorded, the algorithm terminates in line 11. If not, each adjacent node $v$ of $u$ is checked for possible subpath extension $p_{v k}$. Line 16 ensures that $p_{v k}$ is simple. The signal parameters for $p_{v k}$ are updated accordingly to each link block $m$ of link $(u, v)$ in lines 17-39. $p_{v k}$ is inserted into $Q$ in line 40. If $K$ feasible connections do not exist, the recorded connections (less than $K)$ are returned in line 41. 


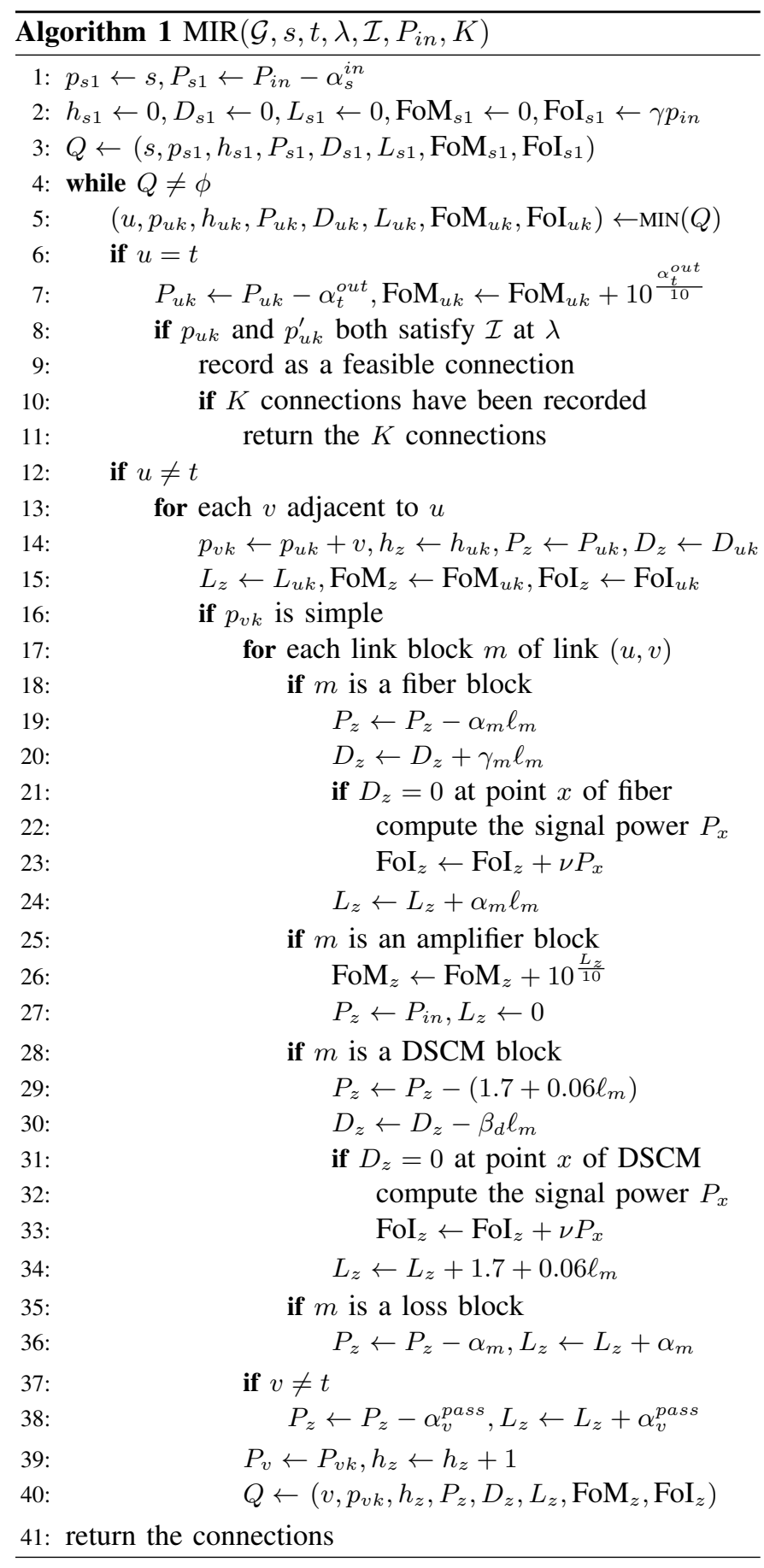

Line 5 takes at most $O\left(k_{\max } N \log \left(k_{\max } N\right)\right)$ time, since $Q$ contains at most $k_{\max } N$ subpaths. Line 8 takes at most $O(E L I)$ time. The for loop in line 13 takes at most $O\left(k_{\max } E\right)$ time, since it is invoked at most $k_{\max }$ times for each side of each link. Hence, the worst-case complexity of the MIR algorithm is $O\left(k_{\max } N E L I \log \left(k_{\max } N\right)+k_{\max } E^{2}\right)$. Since $k_{\max }$ can grow exponentially with the input, the MIR algorithm has an exponential running time. However, a polynomial-time tuneable heuristic for the MIR algorithm can be derived by fixing $k_{\max }$ as done in [14].

\section{B. Phase 2}

In Phase 2, we use the pre-computed $K$ connections of Phase 1 as input to an Integer Linear Programming (ILP) formulation. The ILP returns an optimal assignment of wavelengths for all requests while minimizing $Y$. We also tailored the ILP objective such that requests with $10 \mathrm{Gbps}$ transceivers are assigned starting from low wavelengths while requests with $100 \mathrm{Gbps}$ transceivers are assigned starting from high wavelengths. The separation will reduce the XPM effect between $10 \mathrm{Gbps}$ and $100 \mathrm{Gbps}$ signals.

\section{ILP Constants and Variables:}

$\begin{array}{cl}a_{r k \lambda} & \text { is } 1 \text { if } r \text { uses its } K \text {-th connection and } \lambda \text {; else } 0 \\ b_{r} & \text { is } 1 r \text { has a } 10 \mathrm{Gbps} \text { rate; else } 0 \\ c_{r} & \text { is } 1 r \text { has a } 100 \mathrm{Gbps} \text { rate; else } 0 \\ d_{r k u v} & \text { is } 1 \text { if the } K \text {-th connection of } r \text { uses }(u, v) \text {; else } 0\end{array}$

\section{ILP Objective:}

$$
\operatorname{minimize} \sum_{r \in \mathcal{R}, \kappa \in \mathcal{K}, \lambda \in \mathcal{Y}}\left(\lambda a_{r \kappa \lambda} b_{r}+(Y-\lambda)\left(a_{r \kappa \lambda} c_{r}\right)\right)
$$

\section{ILP Constraints:}

Each request has one connection and one wavelength

$$
\sum_{\kappa \in K, \lambda \in \mathcal{Y}} a_{r \kappa \lambda}=1 \quad \forall r \in \mathcal{R}
$$

One connection is picked from the $K$ connections

$$
\alpha_{r \kappa \lambda} \leq \sum_{u \in \mathcal{N}, v \in \mathcal{N}} d_{\text {rкuv }} \quad \forall \lambda \in \mathcal{Y}, r \in \mathcal{R}, \kappa \in K
$$

A wavelength can only be used once per link

$$
\sum_{r \in \mathcal{R}, \kappa \in K} d_{r \kappa u v} a_{r \kappa \lambda} \leq 1 \quad \forall \lambda \in \mathcal{Y},(u, v) \in \mathcal{E}
$$

Amplifier saturation gain limit

$$
P_{\text {sat }} \geq \sum_{r \in \mathcal{R}, \kappa \in K, \lambda \in \mathcal{Y}} d_{r \kappa u v} a_{r \kappa \lambda} P_{i n}^{r} \quad \forall(u, v) \in \mathcal{E}
$$

\section{Simulation and Analysis}

For our analysis, we use a realistic set of link blocks, one realistic traffic matrix (Traffic Matrix 1) of 195 requests and one projected traffic matrix (Traffic Matrix 2) of 200 requests of the SURFnet7 network [15]. A sample zoom-in view of a link in the SURFnet7 network is illustrated in Figure 3. The other links also consist of a number of different link blocks. Shaded nodes have (R)OADMs, while unshaded nodes have OADMs. Our simulation parameters are given in Table I. The simulation was conducted on an Intel(R) Core i7-4600U $2.1 \mathrm{GHz}$ machine with 16GB RAM memory. 


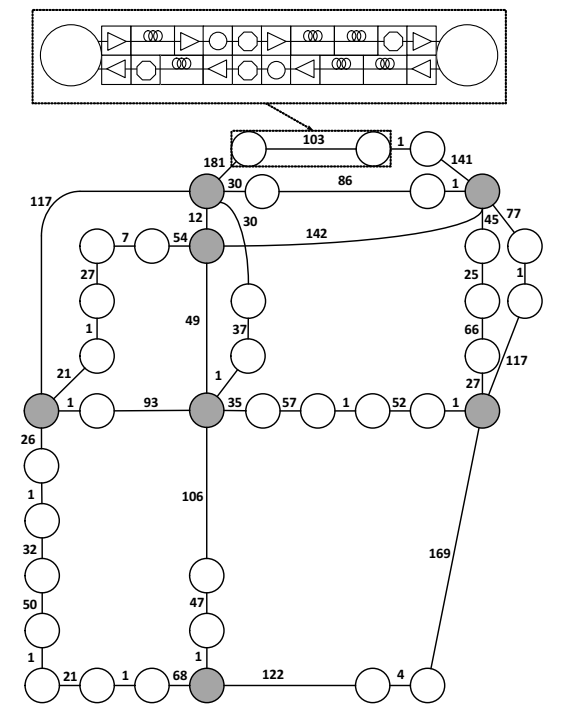

Fig. 3. An example of a zoom-in view of a link in the SURFnet7 network.

TABLE II

Simulation Results (With Phase 2).

\begin{tabular}{|c|c|c|c|c|}
\hline \multirow{2}{*}{ K } & \multicolumn{2}{|c|}{ Traffic Matrix 1 } & \multicolumn{2}{c|}{ Traffic Matrix 2 } \\
\cline { 2 - 5 } & $\begin{array}{c}\text { Needed } \\
\text { Nuvelengths }\end{array}$ & $\begin{array}{c}\text { Running } \\
\text { Time } \\
\text { (minutes) }\end{array}$ & $\begin{array}{c}\text { Wavelengths } \\
\text { Needed }\end{array}$ & $\begin{array}{c}\text { Running } \\
\text { Time } \\
\text { (minutes) }\end{array}$ \\
\hline \hline 1 & 47 & 1.53 & 29 & 1.38 \\
\hline 2 & 33 & 3.72 & 25 & 2.98 \\
\hline 3 & 29 & 6.05 & 25 & 4.08 \\
\hline 4 & 27 & 8.95 & 24 & 5.38 \\
\hline 5 & 27 & 12.18 & 24 & 6.53 \\
\hline
\end{tabular}

TABLE III

Simulation Results (With First-Fit instead of Phase 2),

\begin{tabular}{|c|c|c|c|c|}
\hline \multirow{2}{*}{ K } & \multicolumn{2}{|c|}{ Traffic Matrix 1 } & \multicolumn{2}{c|}{ Traffic Matrix 2 } \\
\cline { 2 - 5 } & $\begin{array}{c}\text { Wavelengths } \\
\text { Needed }\end{array}$ & $\begin{array}{c}\text { Running } \\
\text { Time } \\
\text { (minutes) }\end{array}$ & $\begin{array}{c}\text { Wavelengths } \\
\text { Needed }\end{array}$ & $\begin{array}{c}\text { Running } \\
\text { Time } \\
\text { (minutes) }\end{array}$ \\
\hline \hline 1 & 51 & 0.47 & 32 & 0.18 \\
\hline 2 & 51 & 1.51 & 32 & 1.18 \\
\hline 3 & 51 & 2.58 & 32 & 1.33 \\
\hline 4 & 51 & 3.40 & 32 & 1.65 \\
\hline 5 & 51 & 3.68 & 32 & 1.95 \\
\hline
\end{tabular}

The simulation results are shown in Table II. The optimality of the solution and running time of the two-phase approach can be tuned by adjusting the value of $K$. As $K$ is increased, the solution gradually becomes better at the cost of higher running time. For all the simulated $K$, Phase 1 takes as little as four seconds, and at most one and half minutes, to be solved. Phase 2 then contributes most to the simulation running time.

As an alternative, Phase 2 can be substituted with a variant of the first-fit wavelength assignment approach [16]. In the increasing order of path hop counts of its $K$ connections, each $r \in \mathcal{R}$ is assigned with a bidirectional connection and a wavelength while ensuring that all of the conditions imposed by the ILP of Phase 2 are also satisfied. The simulation results are shown in Table III. Though this approach is fast, the solution is not as good as our Phase 2. The additional candidate paths provided by Phase 1 also do not improve the solution, while significant improvement due to the $K$ connections can be observed when using our two-phase approach.

\section{CONCLUSion}

In this paper, we have proposed a pragmatic approach for quantifying the potential harm of various impairments in realistic networks; a new metric referred to as the Figureof-Impact (FoI) that quantifies the Self-Phase Modulation (SPM) impairment; and a two-phase heuristic for solving the multi-constrained impairment-aware routing and wavelength assignment problem. Our pragmatic approach performs well in a realistic case study of the SURFnet7 network.

\section{ACKNOWLEDGMENT}

This research was supported by the GigaPort 2014 Research on Networks project coordinated by SURFnet.

\section{REFERENCES}

[1] F. Kuipers, A. Beshir, A. Orda, and P. Van Mieghem, "Impairmentaware path selection and regenerator placement in translucent optical networks," in IEEE Int. Conf. Netw. Protocols (ICNP'10), 2010.

[2] B. Ramamurthy, D. Datta, H. Feng, J. P. Heritage, and B. Mukherjee, "Impact of transmission impairments on the teletraffic performance of wavelength-routed optical networks," IEEE J. of Lightwave Technol., vol. 17, no. 10, p. 1713, 1999.

[3] P. Soproni, T. Cinkler, and J. Rak, "Methods for physical impairment constrained routing with selected protection in all-optical networks," Telecommunication Syst., vol. 56, no. 1, pp. 177-188, 2014.

[4] A. Beshir, R. Nuijts, R. Malhotra, and F. Kuipers, "Survivable impairment-aware traffic grooming," in IEEE European Conf. Netw. \& Opt. Commun. (NOC'11), 2011.

[5] H. A. Pereira, D. A. Chaves, C. J. Bastos-Filho, and J. F. Martins-Filho, "OSNR model to consider physical layer impairments in transparent optical networks," Photonic Netw. Commun., vol. 18, no. 2, 2009.

[6] S. Pachnicke, N. Luck, and P. M. Krummrich, "Online physical-layer impairment-aware routing with quality of transmission constraints in translucent optical networks," in IEEE Int. Conf. on Transparent Opt. Netw. (ICTON'09), 2009.

[7] G. Markidis, S. Sygletos, A. Tzanakaki, and I. Tomkos, "Impairment aware based routing and wavelength assignment in transparent long haul networks," in Opt. Netw. Design and Modeling (ONDM'07), 2007.

[8] F. Iqbal, S. Yang, and F. Kuipers, "Energy considerations in EoS-overWDM network configuration," in IEEE Sustainable Internet and ICT for Sustainability Conf. (SustainIT'13), 2013.

[9] S. P. Singh and N. Singh, "Nonlinear effects in optical fibers: Origin, management and applications," Progress In Electromagnetics Res. vol. 73, pp. 249-275, 2007.

[10] Z. Wang and J. Crowcroft, "Quality-of-service routing for supporting multimedia applications," IEEE J. Select. Areas in Commun., vol. 14, no. 7, pp. 1228-1234, 1996.

[11] I. Chlamtac, A. Ganz, and G. Karmi, "Lightpath communications: An approach to high bandwidth optical wan's," IEEE Trans. Commun., vol. 40, no. 7, pp. 1171-1182, 1992.

[12] E. I. Chong, S. Maddila, and S. Morley, "On finding single-source singledestination k shortest paths," J. Comput. Inform., vol. 95, 1995.

[13] P. Van Mieghem and F. Kuipers, "Concepts of exact QoS routing algorithms," IEEE/ACM Trans. Netw., vol. 16, no. 9, pp. 851-864, 2004.

[14] F. Iqbal, J. van der Ham, and F. Kuipers, "Technology-aware multidomain multi-layer routing," Computer Communications, 2015.

[15] [Online]. Available: https://www.surf.nl/en/services-and-products/ surfinternet/network-topology-surfnet7/index.html

[16] H. Zang, J. P. Jue, and B. Mukherjee, "A review of routing and wavelength assignment approaches for wavelength-routed optical wdm networks," Opt. Netw. Mag., vol. 1, no. 1, pp. 47-60, 2000. 